\title{
Quantum phase transitions and topological proximity effects in graphene nanoribbon heterostructures
}

\section{Citation}

Zhang, Gufeng, Xiaoguang Li, Guangfen Wu, Jie Wang, Dimitrie Culcer, Efthimios Kaxiras, and Zhenyu Zhang. 2014. "Quantum Phase Transitions and Topological Proximity Effects in Graphene Nanoribbon Heterostructures." Nanoscale 6 (6): 3259. https://doi.org/10.1039/c3nr05284b.

\section{Permanent link}

http://nrs.harvard.edu/urn-3:HUL.InstRepos:41384063

\section{Terms of Use}

This article was downloaded from Harvard University's DASH repository, and is made available under the terms and conditions applicable to Open Access Policy Articles, as set forth at http:// nrs.harvard.edu/urn-3:HUL.InstRepos:dash.current.terms-of-use\#OAP

\section{Share Your Story}

The Harvard community has made this article openly available. Please share how this access benefits you. Submit a story.

Accessibility 


\title{
Quantum Phase Transitions and Topological Proximity Effects in Graphene Nanoribbon Heterostructures
}

\author{
Gufeng Zhang, ${ }^{1,2,3}$ Xiaoguang Li, ${ }^{2,1,4}$ Guangfen Wu, ${ }^{1,5}$ Jie Wang, ${ }^{1}$ \\ Dimitrie Culcer, ${ }^{1,6}$ Efthimios Kaxiras, ${ }^{7}$ and Zhenyu Zhang ${ }^{1,7}$ \\ ${ }^{1}$ International Center for Quantum Design of Functional Materials(ICQD), \\ Hefei National Laboratory for Physical Sciences at the Microscale, \\ University of Science and Technology of China, Hefei, Anhui, 230026, China \\ ${ }^{2}$ State Key Laboratory of Surface Physics and Department of Physics, Fudan University, Shanghai, 200433, China \\ ${ }^{3}$ Department of Physics, University of California, San Diego, La Jolla, CA 92093, USA \\ ${ }^{4}$ Department of Physics and Astronomy, University of Tennessee, Knoxville, TN 37996, USA \\ ${ }^{5}$ Shenzhen Institutes of Advanced Technology, Chinese Academy of Sciences, Shenzhen, 518055, China \\ ${ }^{6}$ School of Physics, The University of New South Wales, Sydney 2052, Australia \\ ${ }^{7}$ School of Applied Science and Engineering, Harvard University, Cambridge, MA 02138, USA
}

Topological insulators are bulk insulators that possess robust chiral conducting states along their interfaces with normal insulators. A tremendous research effort has recently been devoted to topological insulator-based heterostructures, in which conventional proximity effects give rise to a series of exotic physical phenomena. Here we establish the potential existence of topological proximity effects at the interface between a topological insulator and a normal insulator, using graphene-based heterostructures as prototypical systems. Unlike conventional proximity effects in topological insulator based heterostructures, which refer to various phase transitions associated with the symmetry breaking of specific local order parameters, topological proximity effects describe the rich variety of quantum phase transitions associated with the global properties of the system measured by the location of the topological edge states. Specifically, we show that the location of the topological edge states exhibits a versatile tunability as a function of the interface orientation, the strength of the interface tunnel coupling between a topological graphene nanoribbon and a normal graphene nanoribbon, the spin-orbit coupling strength in the normal graphene nanoribbon, and the width of the system. For zigzag and bearded graphene nanoribbons, the topological edge states can be tuned to be either at the interface or outer edge of the normal ribbon. For armchair graphene nanoribbons, the potential location of the topological edge state can be further shifted to the edge of or within the normal ribbon, to the interface, or diving into the topological graphene nanoribbon. We further show that the topological phase diagram established for the prototypical graphene heterostructures can also explain the intriguing quantum phase transition reported recently in other topological-insulator heterostructures. We also discuss potential experimental realizations of the predicted topological proximity effects, which may pave the way for integrating the salient functionality of topological insulators and graphene in future device applications.

PACS numbers: 73.22.Pr 03.65.Vf 73.40.-c

\section{INTRODUCTION}

The discovery of topological insulators (TIs) has revolutionized our understanding of insulating behavior 111. The appearance of topologically insulating behavior is associated with a topological phase transition [1, 3, 12, 13. For example, when the spin-orbit coupling (SOC) exceeds a critical strength, a band inversion takes place, rendering the entire system topologically nontrivial. Topological phase transitions do not involve symmetry breaking, but entail instead a change in the $Z_{2}$ topological invariant, which may be regarded as a quantity counting the number of Dirac cones. No local order parameters can be defined for a topological phase transition: such TI behavior, which is protected against time-reversal invariant perturbations, is a one-particle phenomenon and is the result of SOC. The carriers at the interface between a TI and a normal insulator are massless Dirac fermions with spin-momentum locking. At the same time, the field of TI also provides a unique platform for studying the interplay between strong SOC and electron-electron interaction effects [14, 15, as manifested by the existence of exotic quantum phase transitions [14].

The robustness of topologically protected surface states may enable hybrid TI heterostructure systems to provide fundamental device improvements as well as potential applications [16]. Recently, by using various TIbased heterostructures, emergent properties of topological surface states have been demonstrated [17-22]. For instance, TI forms a natural heterostructure with ordinary insulators [17, which can be used to manipulate topological states and the bulk band gap. By putting a ferromagnet on a TI, the inverse spin-galvanic effect [18] and giant spin battery effect [19] can be realized. Interesting properties are also found in TI heterostructures with magnetic insulators [20, Mott insulators [21, and $\mathrm{Bi}(111)$ bilayer [22]. In particular, proximity effects in TI heterostructures yield novel phases of matter [23 30. For example, TI/superconductor heterostructures 


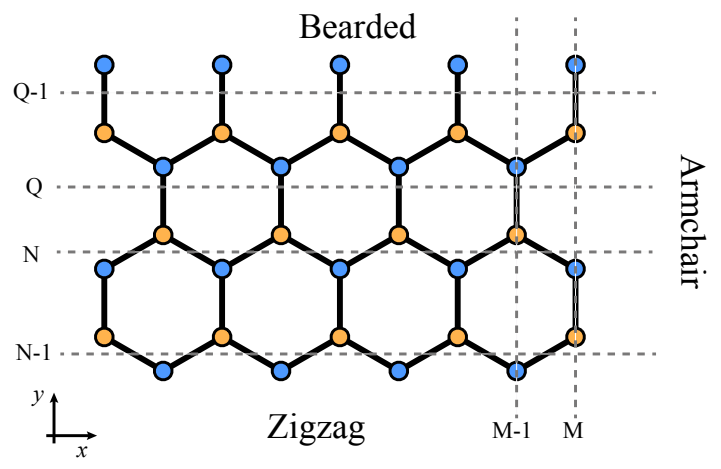

FIG. 1. (Color Online) Graphene ribbon geometry with zigzag, bearded and armchair edges. The dashed lines along the zigzag, armchair, and bearded edges labeled with $N, M$, and $Q$ indicate the $N^{t h}, M^{t h}$ and $Q^{t h}$ unit cell in $y, x$ and $y$ directions, respectively. The width of a ribbon is measured in multiples of the unit cell. Ribbons with zigzag and bearded edged have infinite length in the $x$ direction, and for armchair edges in the $y$ direction.

exhibit a superconducting proximity effect offering the possibility of observing Majorana fermions [23] and the potential realization of non-Abelian topological quantum computation [31. The quantized electromagnetic response in a TI-ferromagnetic material heterostructure is due to a topological magnetoelectric effect [26, 32]. Other novel, technologically important properties have also been demonstrated, such as the enhancement of the catalysis process by robust topological surface states in Au-covered TI 25]. TI-based heterostructures are thus systems of both fundamental and practical importance [23 28]. Harnessing the robust topological surface states entails an accurate understanding and control of their spatial location.

Recent first-principles studies of three-dimensional (3D) TI/normal insulator heterostructures have demonstrated that the spatial location of the surface states can be shifted to the surface of the normal insulator [27, 28, and in a certain parameter range can even be shifted back into the TI bulk [27]. Such studies suggest the possibility of a topological phase transition induced in a normal insulator via topological proximity effects, identified by the topological surface states leaking out into an adjacent material or moving back into the TI. Whereas conventional proximity effects can be described using local order parameters, topological proximity effects involve a topological phase transition for which a local order parameter cannot be defined. In light of this, a practical and highly nontrivial issue to be addressed is the determination of the exact spatial location of the topological surface states in such TI-based heterostructures.

In this paper, we choose graphene as a prototype to explore the general features of the topological proximity effects. Graphene is of great interest, since it has a simple honeycomb lattice structure, while possessing numerous intriguing topological phenomena, such as the in- teger quantum Hall effect [33, anomalous quantum Hall effect [34, and fractional quantum Hall effect [35. In particular, graphene can be turned into a TI once the intrinsic SOC reaches a large enough value [1. Given the ample energy band structure tunability of graphene, it is convenient to use a graphene-based heterostructure as a prototype to systematically investigate the topological proximity effects by considering several crucial parameters of the model system. We expect that the obtained features of topological proximity effects are also suitable for other 2D or 3D TI-based systems, and may be instructive for the design of future TI-based electronic devices.

We explore topological proximity effects on topological phase transitions in an important material class of graphene heterostructures 36 consisting of a topological graphene nanoribbon (GNR) and a gapped normal GNR. Three different interface orientations are investigated: zigzag, bearded, and armchair (Fig. 1). Unlike conventional proximity effects, in which a phase transition is accompanied by symmetry breaking measured by a local order parameter, the topological proximity effects introduced here surrounding topological phase transitions do not involve a symmetry breaking process. Instead of a local order parameter, the measurement of such topological proximity effects is the location of the topological edge states (TESs), which is determined in this study as a function of the interface coupling strength, the SOC strength in the normal GNR, and the orientations of the interface. For three different interface orientations: zigzag, bearded, and armchair, we demonstrate versatile tunabilities in the location of the TES. For zigzag and bearded [37] GNRs, it can be tuned to be either at the interface or the outer edge of the normal GNR. For armchair GNRs, the potential location of the TES is further enriched to be at the edge of or within the normal GNR, at the interface, or diving into the topological GNR. Moreover, the dependence of the TES behaviors on the interface orientation is attributed to the different locations of the Dirac points of the TES in $\boldsymbol{k}$ space for different heterostructures. Taken together, our findings illustrate the conceptual complexity as well as richness of the topological proximity effect at TI-based heterostructures.

This paper is organized as follows: In Sec. II], we introduce the tight binding Kane-Mele model for GNR heterostructures and outline our methodology. In Sec. III, we show the tunability of the spatial location of the TES as some key parameters in zigzag, bearded, and armchair GNR heterostructures. Before concluding in Sec. V] we mainly discuss and explain different TES behaviors for different kinds of interface orientations in Sec. IV], where potential applications and experimental realizations are also presented. 


\section{METHODOLOGY}

We start with the Kane-Mele model [1,2] for GNRs. In the present work, we generalize the systems of interest to explore the topological proximity effects in hybrid GNR heterostructures consisting of a normal and a topological GNRs. In these systems, we focus on two central parameters: the tunnel coupling at the interface between the GNRs, and the strength of the SOC in the normal GNR. We investigate the spatial location of the TES at the interface between the two GNRs for three different interface orientations: zigzag, bearded, and armchair (Fig. 1). These three orientations give rise to qualitatively different graphene band structures, and consequently the proximity effects take qualitatively different forms.

For both the topological and normal GNRs, we use the same tight-binding Hamiltonian as follows, but with different specifications on the parameters:

$$
H=t \sum_{\langle i j\rangle} c_{i}^{\dagger} c_{j}+\sum_{i \in a, b} V_{i} c_{i}^{\dagger} c_{i}+i \lambda_{S O} \sum_{\langle\langle i j\rangle\rangle} c_{i}^{\dagger} \boldsymbol{\sigma} \cdot\left(\boldsymbol{d}_{\boldsymbol{k} \boldsymbol{j}} \times \boldsymbol{d}_{\boldsymbol{i k}}\right) c_{j},
$$

where $c_{i}^{\dagger}\left(c_{i}\right)$ is the electron creation (annihilation) operator on site $i$; $t$ is the nearest-neighbor hopping; $V_{a(b)}$ is the on-site energy for the A(B) sublattice; $\lambda_{S O}$ is the intrinsic SOC connecting next-nearest neighbors; $\boldsymbol{\sigma}$ is the Pauli matrix vector; $i$ and $j$ are two next-nearest neighbor sites, $k$ is their unique common nearest neighbor, and the vector $\boldsymbol{d}_{\boldsymbol{i} \boldsymbol{k}}$ points from $k$ to $i$.

The band structure of graphene can be qualitatively changed by tuning the coupling parameters in Eq. (1). When $V_{a}=-V_{b}=V_{g} / 2$ and $\lambda_{S O}=0$, we can obtain a trivial insulator with the band gap equals to $V_{g}$. On the other hand, in a pristine graphene where we have $V_{g}=0$, a nontrivial insulator can be obtained with the band gap of $6 \sqrt{3} \lambda_{S O}$. Essentially, the competing between $V_{g}$ and $\lambda_{S O}$ determines the topological phase of the graphene. Although the intrinsic SOC in carbon system is too weak to produce a visible nontrivial band gap $(100 \mathrm{mK}$ [1, 3840]), many viable approaches have been proposed to enhance this. For example, the impurity-induced, lattice driven SOC can be of at least the same order of magnitude as the atomic SOC [41; certain heavy non-magnetic adatoms such as indium and thallium can enhance the intrinsic SOC gap up to room temperature 38; random adsorption of adatoms can suppress intervalley scattering, which is detrimental to the topological phase, but does not affect the induced SOC, consequently stabilizing the topological phase in graphene [42].

All GNR heterostructures we discuss consist of a normal and a topological GNR with the same interface orientation. The width of the GNRs is measured in multiples of the unit cell illustrated in Fig. 1. In the topological GNR, we set the SOC strength as a constant $\lambda_{S O}=0.03 t \approx 0.08 \mathrm{eV}$, and the on-site energy $V_{g}=0$. In the normal GNR, we assume a finite $V_{g}$ to open a trivial band gap, and a relatively small $\lambda_{S O}$, which is not large enough to induce a topological phase transition as the GNR is isolated. The interface tunnel coupling $t_{c}$ appears as the nearest-neighbor hopping energy between the normal and topological GNRs; for zigzag GNR heterostructures, the bonds with tunable coupling $t_{c}$ are illustrated by the red lines in Figs. 2(e-h). The heterostrctures are assumed to be infinite in the direction parallel to the interface. We thus solve an effective 1D problem by diagonalizing the Hamiltonian in Eq. 1 to obtain band structures and the corresponding edge states.

\section{RESULTS}

In this section, we report the results of band structure and spatial locations edge states in the GNR heterostructures with different orientations (zigzag, bearded and armchair). In particular, we investigate the way the TES moves in such systems as a result of the interplay of the interface tunneling, the spin-orbit coupling in the normal GNR and the thickness of the normal GNR. Throughout this study, we consider the thickness of topological GNR to be a large enough constant to avoid direct interaction between the topological edge states on the two sides of the structure. Also, we note that for simplicity, we consider a local interface coupling in this paper. Consideration of a continuously changing interface hopping strength gives qualitatively the same results.

\section{A. Zigzag graphene nanoribbon heterostructures}

\section{How interface tunneling affects the position of topological edge states}

We first investigate GNR heterostructures with zigzag edges. The heterostructure consists of a normal GNR with width $W_{n}=3$, and a topological GNR with width $W_{t}=30$. Originally, the normal zigzag GNR possesses gapless edge states 43. To distinguish between the topological and trivial edge states, we open a band gap in the normal GNR by adding the different on-site energy for the two sublattices. In Figs. 2(a-d), we exhibit a series of band structures of systems with the different interface coupling $t_{c}$, while the fixed on-site energy $V_{a}=-V_{b}=0.1 t$ and SOC strength $\lambda_{S O}=0$ in the normal GNR. $E_{1}$ and $E_{2}$ represent the energy of trivial edge states, which locate separately at the edges of the normal GNR, and the Dirac points (denoted as $D_{1}$ and $D_{2}$ ) describing the band crossing of the TESs to emphasize the linear dispersion around the corresponding $k$ point. We also show spin orientations near the Fermi level in Figs. 2(b-d) with spin up in blue and spin down in red. Based on our definition, the electrons of $E_{2}$ and $D_{1}$ are located near the interface between the normal and topological GNRs, and therefore are more sensitive to the tunnel coupling $t_{c}$. As we gradually increase $t_{c}$ as illustrated in Fig. 2 from left to right, both the energies and spatial locations of these edge states will change ac- 


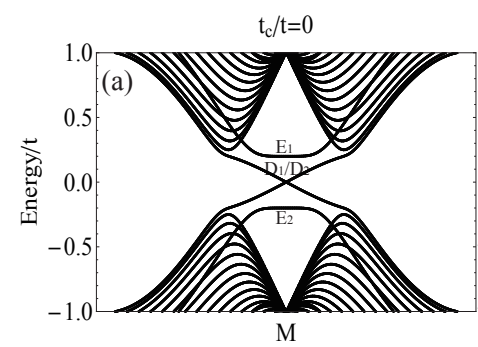

(e)

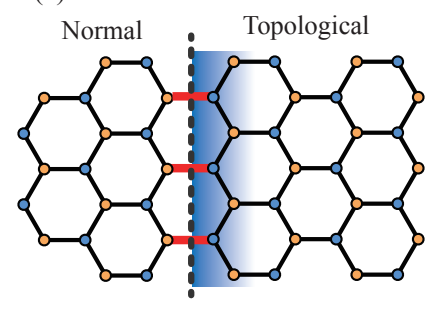

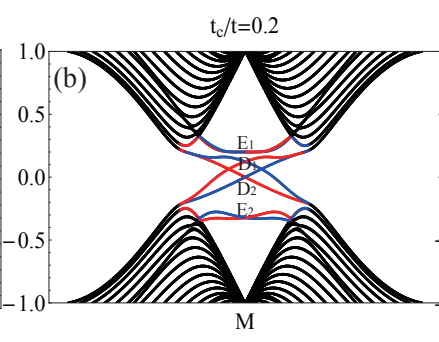

(f)

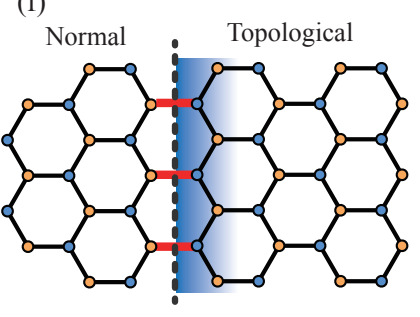

$\mathrm{t}_{\mathrm{c}} / \mathrm{t}=0.32$

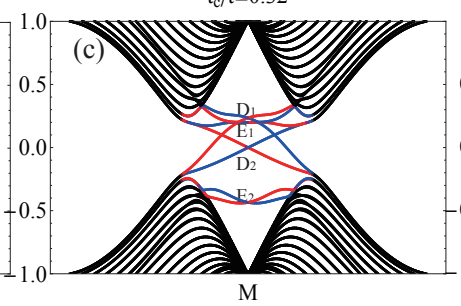

(g)

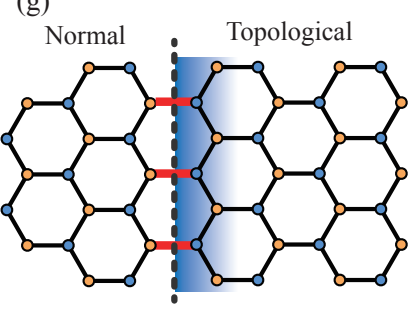

$\mathrm{t}_{\mathrm{c}} / \mathrm{t}=0.45$

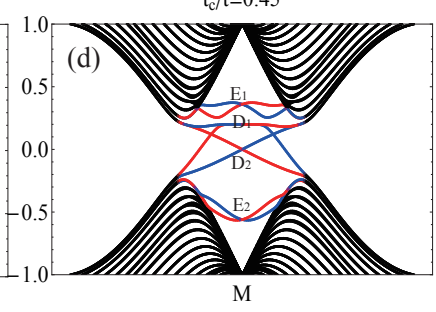

(h)

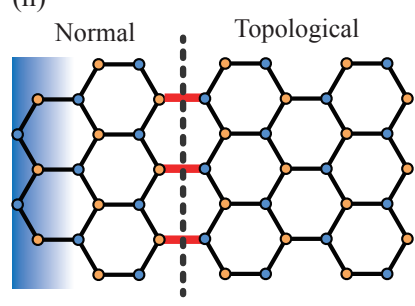

FIG. 2. (Color Online) Band structures of zigzag edge GNR heterostructures $\left(W_{n}=3, W_{t}=30\right)$ for different tunnel coupling strengths (a-d), and the corresponding spatial locations of higher energy Dirac points (denoted as $D_{1}$ in (a-d)) are shown by blue areas in (e-h). Different spin orientations are shown as spin up (blue) and spin down (red) near Fermi levels in band structures. The interface tunnel coupling $t_{c}=0$ in $(\mathrm{a})(\mathrm{e}), t_{c}=0.2 t$ in $(\mathrm{b})(\mathrm{f}), t_{c}=0.32 t$ in $(\mathrm{c})(\mathrm{g}), t_{c}=0.45 t$ in $(\mathrm{d})(\mathrm{h})$. For the normal GNR, $V_{g}=0.1 t$ and $\lambda_{S O}=0$; for the topological GNR, $V_{g}=0$ and $\lambda_{S O}=0.03 t$. $E_{1}$ and $E_{2}$ : the bands of the trivial edge states originated from the normal GNR. $D_{2}$ : the other Dirac point whose spatial location is far away from the interface. Dashed lines in $(\mathrm{e}-\mathrm{h})$ indicate the interface of the heterostructures, and the red bonds show the interface tunnel coupling $\left(t_{c}\right)$.

cordingly. To see the evolution of the states clearly, we analyze the transition as follows:

- $t_{c} / t=0$ (Figs. 2(a)(e)). The two GNRs are detached, and the two Dirac points are degenerate. The state $D_{1}$ is located at the interface of the heterostructure, as shown by the blue area in Fig.2(e).

- $t_{c} / t=0.2$ (Figs. 2(b)(f)). As $t_{c}$ increases, the interaction between bands $D_{1}$ and $E_{2}$ results in the energy of $D_{1}$ increasing and that of $E_{2}$ decreasing. But the spatial location of $D_{1}$ does not change.

- $t_{c} / t=0.32$ (Figs. 2(c)(g)). The energy of $D_{1}$ increases to slightly exceed $E_{1}$. The spatial location of $D_{1}$ stays at the interface.

- $t_{c} / t=0.45$ (Figs. 2(d)(h)). The topological phase transition happens when the bands $E_{1}$ and $D_{1}$ detach. Now $D_{1}$ becomes the flat band with energy lower than $E_{1}$, and its spatial location moves to the outer edge of the normal GNR (Fig. 2(h)).

After the phase transition, further increasing $t_{c}$ will not change the energy and spatial location of $D_{1}$, but will keep enlarging the energy of $E_{1}$ and reducing the energy of $E_{2}$ toward bulk states, implying that these two states are spatially more close now. In the whole process, the energy of $D_{2}$ does not change, because its spatial location is far away from the interface. For very large $t_{c}, E_{1}$ and $E_{2}$ are mixed with bulk states, and we can see only two TESs located separately at two edges of the whole heterostructures. The shift of TESs from the interface

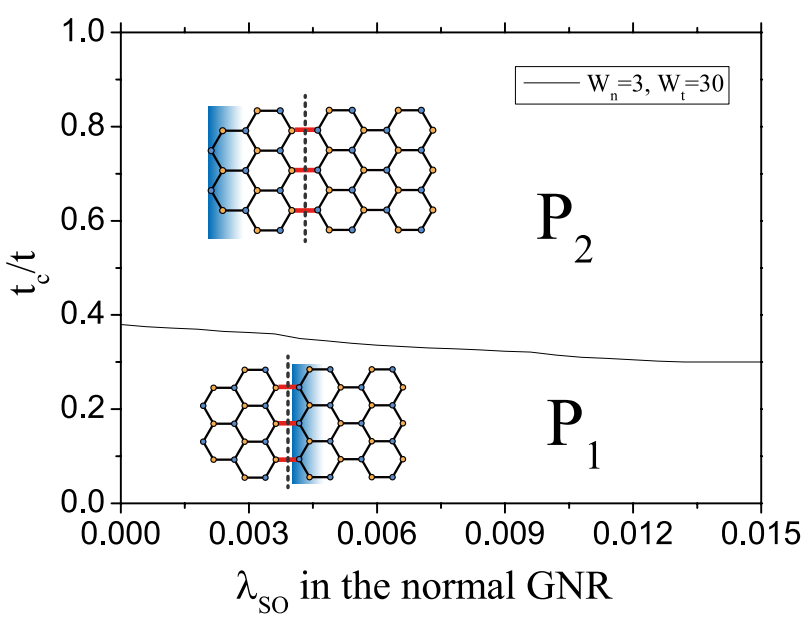

FIG. 3. (Color Online) Phase diagram for zigzag GNR heterostructures, spanned by the tunnel coupling $\left(t_{c}\right)$ and SOC in the normal GNR. The black solid line indicates the boundary of $P_{1}$ and $P_{2}$ phases with $W_{n}=3, W_{t}=30$. Insets are the illustrations of the spatial locations of the TESs. $P_{1}$ phase: the TES is located at the interface. $P_{2}$ phase: the TES is located at the outer edge of the normal GNR.

to the outer edge of the normal GNR indicates that the heterostructure in its entirety becomes an expanded 2D TI via the topological proximity effect. 


\section{How spin-orbit coupling affects the position of topological edges states}

Qualitatively similar pictures are observed when we include a finite SOC in the normal GNR. Under the influence of both the tunnel coupling and SOC, the system still has two phases. As shown in the phase diagram Fig. 3, the phase transition occurs at the boundary of the $P_{1}$ and $P_{2}$ areas. Specifically, for given values of $t$ and $\lambda_{S O}, t_{c}$ separating the two phases decreases monotonically as the SOC in the normal GNR increases. In other words, the SOC in the normal GNR helps the transition happen. We can understand this physical picture by considering the limiting case when we apply a large enough SOC in the normal GNR to induce the topological phase transition without the presence of other topological proximities. In this case, the TES will not appear at the interface ( $P_{1}$ phase) for any finite $t_{c}$, but at the edges of the whole heterostructure $\left(P_{2}\right.$ phase), because both GNRs are topologically nontrivial.

\section{Whole phase diagram of the position of topological edge states}

Furthermore, we discuss the way the width of the normal GNR affects the phase transition. Fig. 4 shows the phase diagram for normal GNR with different widths $W_{n}=3,10,15,20,25$. We see that for the same SOC in the normal GNR, the wider one requires a larger tunnel coupling $t_{c}$ to induce the transition from $P_{1}$ to $P_{2}$. This is because the phase transition essentially needs the coupling between the states at the interface and outer edge of the normal GNR, and this coupling becomes weaker as the width increases. So a larger tunnel coupling $t_{c}$ is required to propagate the TESs to further position. In an limiting case where the normal ribbon has infinite width, topological proximity effect will not occur for any finite tunnel coupling $t_{c}$.

In summary for the zigzag GNR heterostructures, two phases exist with different tunnel coupling and SOC in the normal GNR: the TES may be located at the interface or at the outer edge of the normal GNR.

\section{B. Bearded graphene nanoribbon heterostructures}

We now consider bearded edge graphene nanoribbon 37 heterostructures. Similar to zigzag GNR, a bearded GNR has gapless trivial edge states. After turning on the SOC, a Dirac point emerges at $\Gamma$ point $(\mathrm{k}=0)[$ Fig. $8(\mathrm{~b})]$. We show the topological phase diagram of the bearded edge GNR heterostructure in Fig. 5. The system consists of a normal GNR with width $W_{n}=3$ and topological GNR $W_{t}=30$. As one increases the interface tunnel strength $\left(t_{c}\right)$ between the heterostructures, the TES moves from the interface ( $P_{1}$ phase) to the outer

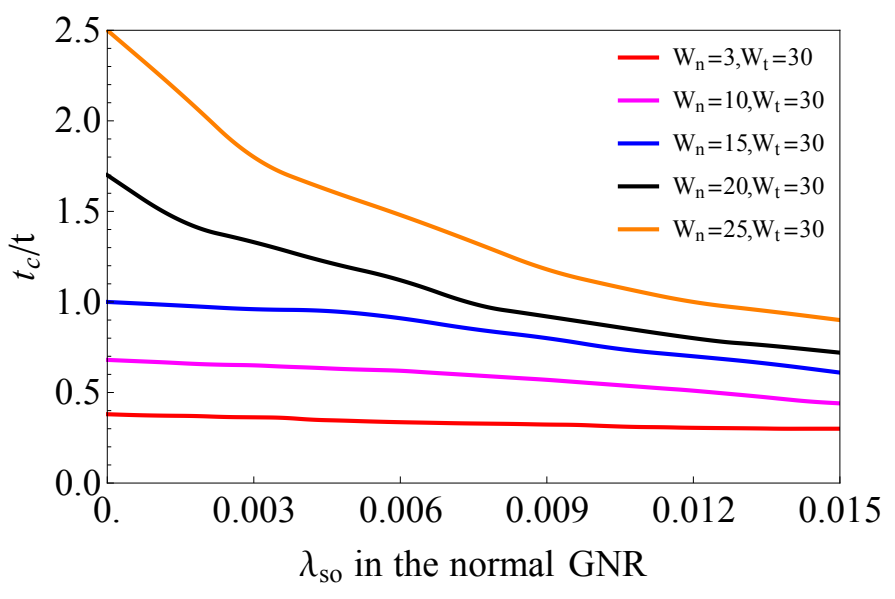

FIG. 4. (Color Online) Phase diagram for zigzag GNR heterostructures with different normal GNR widths, spanned by the tunnel coupling $\left(t_{c}\right)$ and SOC in the normal GNR. The lines indicate the boundary of the $P_{1}$ and $P_{2}$ phases, which are defined the same as in Fig. 3. We use different colors for various widths, as shown in the inset.

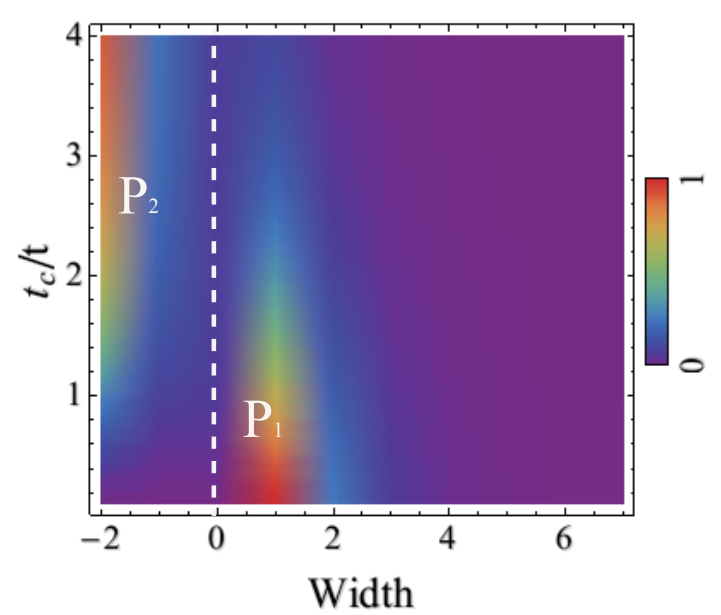

FIG. 5. (Color online) Phase diagram of the TES location without SOC in the normal GNR. The heterostuctures consist of a normal GNR with width $W_{n}=3$ and a topological GNR with width $W_{t}=30$. The dashed line indicates the interface of the heterostructures. $P_{1}$ phase: the TES is located at the interface. $P_{2}$ phase: the TES is located at the outer edge of the normal GNR.

edge of the normal ribbon $\left(P_{2}\right.$ phase $)[$ Fig. 5. Furthermore, switching on the SOC in the normal GNR still preserves the two phases for the TES. The influence of SOC in normal GNR on the topological proximity effect is the same as in the zigzag case. 

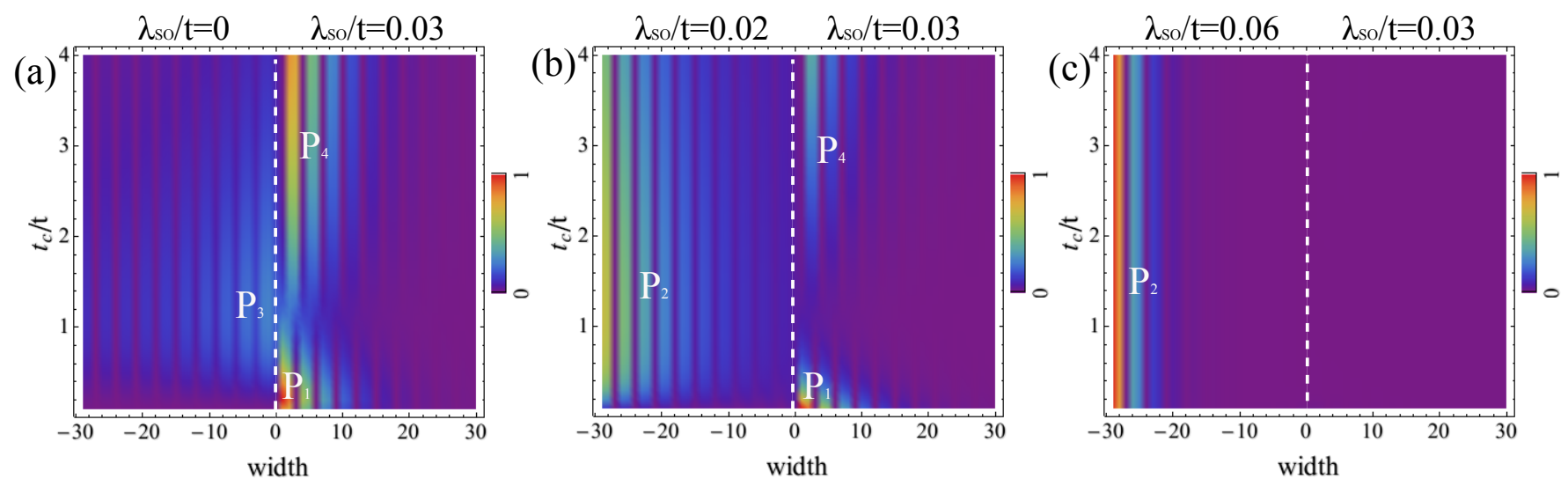

FIG. 6. (Color online) Phase diagram of the TES location for armchair GNR heterostructures with a normal GNR width $W_{n}=30$ on the left and a topological GNR width $W_{t}=60$ on the right. The SOC strength $\lambda_{S O}=0.03 t$ in the topological GNR and is $0(\mathrm{a}), 0.02 t(\mathrm{~b})$ and $0.06 t(\mathrm{c})$ in the normal GNR. The on-site energy in the normal GNR is $V_{g}=0.03 t$. The dashed lines indicate the interface location. Four phases appear for the TES as the tunnel coupling $\left(t_{c}\right)$ increases: $P_{1}$ phase: the TES is located at the interface; $P_{2}$ phase: the TES at the outer edge of the normal GNR; $P_{3}$ phase: the TES in the bulk of the normal GNR; $P_{4}$ phase: the TES is located one unit cell into the topological GNR.

\section{Armchair graphene nanoribbon heterostructures}

Armchair edge GNR heterostructures differ a lot from both zigzag and bearded edge systems in that a pristine (i.e. without SOC) armchair GNR does not have trivial edge states [43]. We first investigate the spatial location of the TES as the tunnel coupling $t_{c}$ increases for the fixed SOC in the normal GNR. Fig. 6 displays our calculation results for three SOC values in the normal GNR: (a) no SOC; (b) moderate $\operatorname{SOC}\left(\lambda_{S O} / t=0.02\right)$ and (c) strong $\operatorname{SOC}\left(\lambda_{S O} / t=0.06\right)$, which is large enough to induce topological phase transition in the normal GNR at $t_{c}=0$. In contrast with the zigzag and bearded cases, the armchair GNR heterostructure shows two additional phases in the strong tunnel coupling regime, $P_{3}$, where the density of the TES peaks in the normal GNR, and $P_{4}$, where the TES is re-located one unit cell back inside the topological GNR. With no SOC in the normal GNR (a), the location of the TES shifts from $P_{1}$ through $P_{3}$ to $P_{4}$ as the coupling increases, while for a moderate SOC (b), the TES can move to the outer edge of the normal GNR through $P_{2}$ instead of $P_{3}$ during the evolution. In the limiting case of the strong SOC, the original normal GNR becomes topologically nontrivial. Thus the whole system becomes a 2D TI. With any finite $t_{c}$, the TES will only exist at the boundary of the whole system as shown in Fig. 6. We note that neither the $P_{3}$ nor the $P_{4}$ phase exists for the zigzag or bearded GNR, and the appearance of the $P_{4}$ phase is consistent with $a b$ initio work reported recently [27.

Next, we investigate a series of phase diagram by considering the widths of the normal GNR. Fig. 7 displays the phase diagrams as a function of the tunnel coupling and SOC in the normal GNR for the heterostructures with various normal GNR width $W_{n}$ and fixed topological
GNR width $W_{t}=60$. Generally speaking, as $t_{c}$ increases, we see three possible evolution routines for a fixed SOC in the normal GNR: $P_{1} \rightarrow P_{2} \rightarrow P_{4}, P_{1} \rightarrow P_{3} \rightarrow P_{4}$, and $P_{2}$, which correspond to the three subfigures in Fig. 6 . From Fig. 7, we see clearly that the phase space of $P_{2}$ extends as the width increases. For a wider normal GNR and large enough SOC, the TESs will not move one unit cell into the topological GNR ( $P_{4}$ phase), but continue to reside at the outer edge of the normal GNR ( $P_{2}$ phase). In such a case, the whole heterostructure acts as a topological insulator which has the TESs on both sides.

\section{DISCUSSION}

The movement of the TES to different locations is a manifestation of the complexity of the topological proximity effect. As the interface tunnel coupling becomes stronger, it gets easier for the SOC on the topological side of the junction to leak into the part without SOC, which gives rise to an effective SOC in the normal GNR. However, the phase diagram of the TES locations is different for of the GNR heterostructures with different orientations. The existence of the trivial edge states in zigzag and bearded heterostructures plays an important role in causing this qualitative difference. Fig. 8 displays the band structures of the isolated topological GNRs with different edge orientations. We note that the Dirac points for the armchair, bearded and zigzag edge GNRs appear at different $k$ points. For the zigzag (bearded) edge GNR, the Dirac point at $\mathrm{M}(\Gamma)$ point is energetically far away from the bulk states. Consequently, the TES at the $\mathrm{M}(\Gamma)$ points prefers to mix with the edge states in normal GNR, which is energetically close to the TES, therefore this rules out the existence of the $P_{3}$ and $P_{4}$ phases. However, for the armchair edge GNR, the energy of the Dirac 

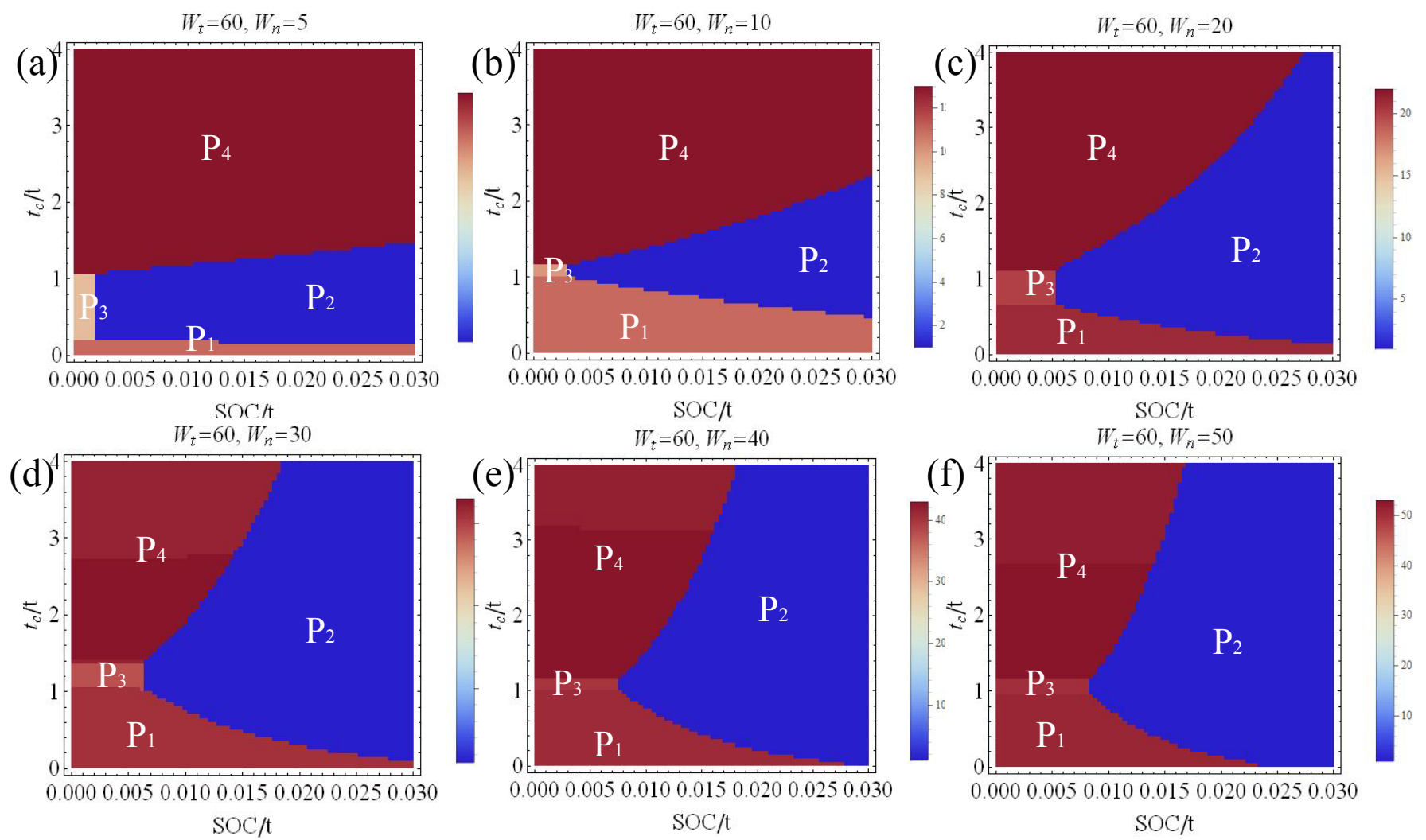

FIG. 7. (Color online) Phase diagram for the armchair GNR heterostructures with a topological GNR width $W_{t}=60$ and a normal GNR width $W_{n}=5(\mathrm{a}), W_{n}=10(\mathrm{~b}), W_{n}=20(\mathrm{c}), W_{n}=30(\mathrm{~d}), W_{n}=40(\mathrm{e}), W_{n}=50$ (f). All diagrams are spanned by the tunnel coupling $\left(t_{c} / t\right)$ and SOC in the normal GNR. The four phases are defined same as in Fig. 6

point is close to the bulk bands and the trivial edge state does not exist. As a consequence, it is possible for the TES to interact with the bulk states and move into the bulk, providing the tow more phases $P_{3}$ and $P_{4}$ related to the bulk states.

In reality, most known TIs have similar band structures to armchair GNRs, namely, the Dirac points of the TES are located at the same $k$ point as the bulk band gap (Fig. 8(a)). We thus expect that the phase diagram of the armchair GNR heterostructure is also applicable to the similar TI-based heterostructures. In a recent $a b i n i-$ tio study, Wu et al [27] have observed in 3D TI/normal insulator heterostructures that the spatial location of the topological surface states can be located at the interface, shifted to the surface of the normal insulator, or back into the TI bulk, which corresponds to $P_{1}, P_{2}$, and $P_{4}$ in our phase diagram, respectively. On the other hand, the new discovered $P_{3}$ phase in this study can be expected in the TI/normal insulator heterostructures with a relatively thick normal insulator layer. For the zigzag GNR heterostructures, we also expect an analogy between the prototypical graphene system and other 3D TI/normal insulator heterostructures with the similar band structures.

At this point, it is worthwhile to emphasize that conventional proximity effects involve the order parameter of a broken-symmetry phase of a host material leaking into an adjacent material, which is driven into a broken symmetry state of the host material as well. In the topological proximity effects demonstrated here, modulations of the SOC and interface tunnel coupling shift the boundary between a normal insulator and a TI, accompanied by topological phase transitions. A fascinating feature of the topological proximity effects is the dual-proximity nature: the location of the TES can be switched back and forth between the two materials, including placing the topological phase boundary inside an otherwise structurally homogenous material such as the normal insulator or the TI. One can regard a conventional proximity effect as a spatial extension of a broken symmetry state. In contrast, the topological proximity effect refers to inducing chiral surface states in an adjacent material as well as controlling their location, again, without symmetry breaking.

Aside from the conceptual advances, the present study may also offer new opportunities in developing spintronic devices and quantum computing. For example, the systems proposed in this work can be used to induce chiral spin-polarized states in a 2D graphene slab at will, which constitutes an ON/OFF switch based on the TES, and can be regarded as a qubit. Such a switch can be controlled by the SOC or the tunnel coupling. As another 

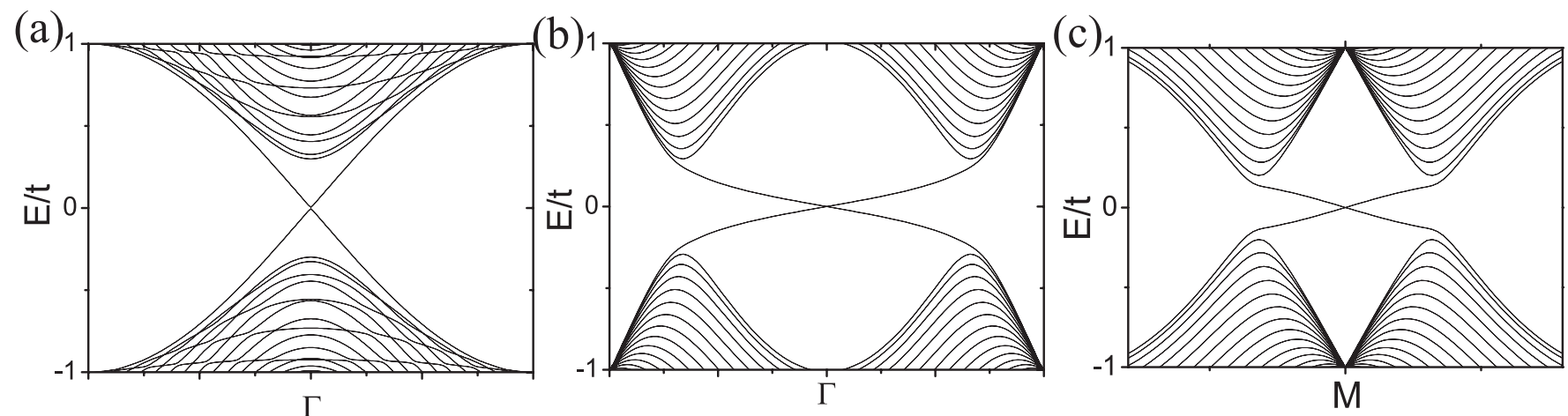

FIG. 8. (Color online) Band structures of an armchair (a), bearded (b), and zigzag (c) topological GNRs with the widths $W_{t}=30$.

example, we can further regard the TES as a current loop, and join a square slab of a topological GNR with a square slab of a normal GNR. Whereas the topological side is always in the ON state, the state of the normal insulator side can be modulated. A spin polarization may then be induced in this qubit by coupling it to a ferromagnet, which would enable control of the quantum anomalous Hall effect, and would also serve as a spin injector into the normal GNR.

Although the predicted topological proximity effects are not limited for the graphene systems, as a very promising material for future electronic devices, we would like to briefly discuss the potential experimental realization of the topological proximity effects predicted in graphene. Firstly, as we mentioned before, even though the intrinsic SOC in pristine graphene is commonly known to be quite weak, many approaches have been proposed to enhance it 38, 41, 42. Recent experiment progresses 45. reported colossal enhancement of SOC to $2.5 \mathrm{meV}$ in weakly hydrogenated graphene. Secondly, the coupling between a normal and a topological GNR can be effectively tuned by substrate steps, as demonstrated recently in Ref. [4], where the resistance from the steps on the $\mathrm{SiC}$ substrate was found to rise due to the abrupt variation in potential and doping as the graphene extends over a step. Lastly, the recently predicted $2 \mathrm{D}$ organic topological insulators [46, 47] with a hexagonal lattice and much larger SOC would offer another platform to implement the topological proximity effect predicted here. These and other alternative candidate structural systems may therefore provide test grounds for physical realization of topological phase transitions.

\section{CONCLUSIONS}

We have demonstrated the existence of topological proximity effects in GNR heterostructures consisting of a normal and a topological GNR under a variety of experimentally relevant circumstances. For different types of edges - zigzag, bearded, and armchair - the location of the TES is a function of the interface tunnel coupling, the SOC strength in the normal GNR and also the width of the normal GNR, demonstrating a rich quantum phase diagram. These findings pave the way for designing next-generation quantum devices that integrate the functionality of graphene and TI. We also stress that the novel topological proximity effects demonstrated here using the prototypical systems of GNR heterostructures are conceptually also applicable to heterostructures consisting of a normal insulator and a 3D TI [17, 27, 28].

\section{ACKNOWLEDGMENTS}

This work was supported by NSF of China (Grant Nos. 91021019 and 11034006), National Basic Research Program of China (Grant No. 2011CB921801), USDOE (Grant No. DE-FG03-02ER45958), and USNSF (Grant No. 0906025).
[1] C. L. Kane, and E. J. Mele, Quantum Spin Hall Effect in Graphene, Phys. Rev. Lett. 95, 226801 (2005).

[2] C. L. Kane, and E. J. Mele, $Z_{2}$ Topological Order and the Quantum Spin Hall Effect, Phys. Rev. Lett. 95, 146802 (2005)

[3] B. A. Bernevig, T. L. Hughes, and S. C. Zhang, Quantum Spin Hall Effect and Topological Phase Transition in
HgTe Quantum Wells, Science 314, 1757 (2006).

[4] M. König, S. Wiedmann, C. Brüne, A. Roth, H. Buhmann, L. W. Molenkamp, X. L. Qi, and S. C. Zhang, Quantum Spin Hall Insulator State in HgTe Quantum Wells, Science 318, 766 (2007).

[5] L. Fu, C. L. Kane, and E. J. Mele, Topological Insulators in Three Dimensions, Phys. Rev. Lett. 98, 106803 (2007). 
[6] J. E. Moore, and L. Balents, Topological invariants of time-reversal-invariant band structures, Phys. Rev. B 75, 121306 (2007).

[7] D. Hsieh, D. Qian, L. Wray, Y. Xia, Y. S. Hor, R. J. Cava, and M. Z. Hasan, A topological Dirac insulator in a quantum spin Hall phase, Nature (London) 452, 970 (2008).

[8] Y. Xia, D. Qian, D. Hsieh, L. Wray, A. Pal, H. Lin, A. Bansil, D. Grauer, Y. S. Hor, R. J. Cava, and M. Z. Hasan, Observation of a large-gap topological-insulator class with a single Dirac cone on the surface, Nature Phys. 5, 398 (2009).

[9] M. Z. Hasan, and C. L. Kane, Colloquium: Topological insulators, Rev. Mod. Phys. 82, 3045 (2010).

[10] X. L. Qi, and S. C. Zhang, Topological insulators and superconductors, Rev. Mod. Phys. 83, 1057 (2011).

[11] H. J. Zhang, C. X. Liu, X. L. Qi, X. Dai, Z. Fang, and S. C. Zhang, Topological insulators in $\mathrm{Bi}_{2} \mathrm{Se}_{3}, \mathrm{Bi}_{2} \mathrm{Te}_{3}$ and $\mathrm{Sb}_{2} \mathrm{Te}_{3}$ with a single Dirac cone on the surface, Nature Phys. 5, 438 (2009).

[12] M. Kim, C. H. Kim, H. Kim, and J. lhm, Topological quantum phase transitions driven by external electric fields in $\mathrm{Sb}_{2} \mathrm{Te}_{3}$ thin films, Proc. Natl. Acad. Sci. U.S.A. 109, 671 (2011).

[13] Su-Yang Xu, Y. Xia, L. A. Wray, S. Jia, F. Meier, J. H. Dil, J. Osterwalder, B. Slomski, A. Bansil, H. Lin, R. J. Cava, and M. Z. Hasan, Topological Phase Transition and Texture Inversion in a Tunable Topological Insulator, Science 332, 560 (2011).

[14] D. Pesin, and L. Balents, Mott physics and band topology in materials with strong spinCorbit interaction, Nature Phys. 6, 376, (2010).

[15] D. Culcer, Linear response theory of interacting topological insulators, Phys. Rev. B 84, 235411 (2011).

[16] D. Kong, and Y. Cui, Opportunities in chemistry and materials science for topological insulators and their nanostructures, Nature Chem. 3, 845, (2011).

[17] J. A. Hutasoit, and T. D. Stanescu, Induced spin texture in semiconductor/topological insulator heterostructures, Phys. Rev. B 84, 085103 (2011); K. Nakayama, K. Eto, Y. Tanaka, T. Sato, S. Souma, T. Takahashi, K, Segawa, and Y. Ando, Manipulation of Topological States and the Bulk Band Gap Using Natural Heterostructures of a Topological Insulator, Phys. Rev. Lett. 109, 236804 (2012); J. Kim, J. Kim, K. Kim, and S. Jhi, Topological phase transition in Dirac fermionic heterostructures, arXiv:1206.2953

[18] I. Garate, and M. Franz, Inverse Spin-Galvanic Effect in the Interface between a Topological Insulator and a Ferromagnet, Phys. Rev. Lett. 104, 146802 (2010).

[19] F. Mahfouzi, B. K. Nikoli, S.-H. Chen, and C.-R. Chang, Microwave-driven ferromagnetCtopological-insulator heterostructures: The prospect for giant spin battery effect and quantized charge pump devices, Phys. Rev. B 82, 195440 (2010).

[20] W. D. Luo, and X.-L. Qi, Massive Dirac surface states in topological insulator/magnetic insulator heterostructures, Phys. Rev. B 87, 085431 (2013); S. V. Eremeev, V. N. Men'shov, V. V. Tugushev, P. M. Echenique, and E. V. Chulkov, Magnetic proximity effect at the $3 D$ topological insulator/magnetic insulator interface, arXiv:1304.1275v1.

[21] S. Ueda, N. Kawakami, and M. Sigrist, Proximity effects in a topological-insulator/Mott-insulator heterostructure,
arXiv:1303.2781

[22] T. Hirahara, G. Bihlmayer, Y. Sakamoto, M Yamada, H. Miyazaki, S. Kimura, S. Blügel, and S. Hasegawa, Interfacing $2 D$ and $3 D$ Topological Insulators: Bi(111) Bilayer on $\mathrm{Bi}_{2} \mathrm{Te}_{3}$, Phys. Rev. Lett. 107, 166801 (2011); Z. F. Wang, M.-Y Yao, W. M. Ming, L. Miao, F. F. Zhu, C. H. Liu, C. L. Gao, D. Qian, J.-F. Jia, and F. Liu, Creation of helical Dirac fermions by interfacing two gapped systems of ordinary fermions, Nat. Commun. 4, 1384 (2013).

[23] L. Fu, and C. L. Kane, Superconducting Proximity Effect and Majorana Fermions at the Surface of a Topological Insulator, Phys. Rev. Lett. 100, 096407 (2008).

[24] T. D. Stanescu, J. D. Sau, R. M. Lutchyn, and S. Das Sarma, Proximity effect at the superconductorCtopological insulator interface, Phys. Rev. B 81, 241310(R) (2010).

[25] H. Chen, W. G. Zhu, D. Xiao, and Z. Y. Zhang, CO Oxidation Facilitated by Robust Surface States on AuCovered Topological Insulators, Phys. Rev. Lett. 107, 056804 (2011).

[26] X.-L. Qi, R. D. Li, J. D. Zang, and S. C. Zhang, Inducing a Magnetic Monopole with Topological Surface States, Science 323, 1184 (2009).

[27] G. F. Wu, H. Chen, Y. Sun, X. G. Li, P. Cui, C. Franchini, J. L. Wang, X. Q. Chen, and Z. Y. Zhang, Tuning the vertical location of helical surface states in topological insulator heterostructures via dual-proximity effects, Sci. Rep. 3, 1233 (2013).

[28] Q. F. Zhang, Z. Y. Zhang, Z. Y. Zhu, U. Schwingenschlögl, and Y. Cui, Exotic Topological Insulator States and Topological Phase Transitions in $\mathrm{Sb}_{2} \mathrm{Se}_{3}-\mathrm{Bi}_{2} \mathrm{Se}_{3}$ Heterostructures, ACS Nano, 6(3), 2345 (2012).

[29] D. Culcer, Transport in three-dimensional topological insulators: Theory and experiment, Physica E 44, 860 (2012).

[30] Z. Fang, N. Nagaosa, K. S. Takahashi, A. Asamitsu, R. Mathieu, T. Ogasawara, H. Yamada, M. Kawasaki, Y. Tokura, and K. Terakura, The Anomalous Hall Effect and Magnetic Monopoles in Momentum Space, Science 302, 92 (2003).

[31] C. Nayak, S. H. Simon, A. Stern, M. Freedman, and S. Das Sarma, Non-Abelian anyons and topological quantum computation, Rev. Mod. Phys. 80, 1083 (2008).

[32] X.-L. Qi, T. L. Hughes, and S. C. Zhang, Topological field theory of time-reversal invariant insulators, Phys. Rev. B. 78, 195424 (2008).

[33] Y. Zhang, Y.-W. Tan, H. L. Stormer, and P. Kim, Experimental observation of the quantum Hall effect and Berry's phase in graphene, Nature (London) 438, 201 (2005); K. S. Novoselov, Z. Jiang, Y. Zhang, S. V. Morozov, H. L. Stormer, U. Zeitler, J. C. Maan, G. S. Boebinger, P. Kim, and A. K. Geim, Room-Temperature Quantum Hall Effect in Graphene, Science 315, 1379 (2007).

[34] Z. H. Qiao, S. A. Yang, W. X. Feng, W.-K. Tse, J. Ding, Y. G. Yao, J. Wang, and Q. Niu, Quantum anomalous Hall effect in graphene from Rashba and exchange effects, Phys. Rev. B 82, 161414 (2010).

[35] K. I. Bolotin, F. Ghahari, M. D. Shulman, H. L. Stormer, and P. Kim, Observation of the fractional quantum Hall effect in graphene, Nature (London) 462, 196 (2009).

[36] O. Shevtsov, P. Carmier, C. Petitjean, C. Groth, D. Carpentier, and X. Waintal, Graphene-Based Heterojunc- 
tion between Two Topological Insulators, Phys. Rev. X 2, 031004 (2012).

[37] M. Fujita, K. Wakabayashi, K. Nakada, and K. Kusakabe, Peculiar Localized State at Zigzag Graphite Edge, J. Phys. Soc. Jpn. 65, 1920 (1996).

[38] C. Weeks, J. Hu, J. Alicea, M. Franz, and R. Q. Wu, Engineering a Robust Quantum Spin Hall State in Graphene via Adatom Deposition, Phys. Rev. X 1, 021001 (2011).

[39] H. Min, J. E. Hill, N. A. Sinitsyn, B. R. Sahu, L. Kleinman, and A. H. MacDonald, Intrinsic and Rashba spinorbit interactions in graphene sheets, Phys. Rev. B 74, 165310 (2006).

[40] Y. G. Yao, F. Ye, X.-L. Qi, S.-C. Zhang, and Z. Fang, Spin-orbit gap of graphene: First-principles calculations, Phys. Rev. B 75, 041401(R) (2007).

[41] A. H. Castro Neto, and F. Guinea, Impurity-Induced Spin-Orbit Coupling in Graphene, Phys. Rev. Lett. 103, 026804 (2009).

[42] H. Jiang, Z. H. Qiao, H. W. Liu, J. R. Shi, and Q. Niu, Stabilizing Topological Phases in Graphene via Random
Adsorption, Phys. Rev. Lett. 109, 116803 (2012).

[43] K. Wakabayashi, M. Fujita, H. Ajiki, and M. Sigrist, Electronic and magnetic properties of nanographite ribbons, Phys. Rev. B 59, 8271 (1999).

[44] T. Low, V. Perebeinos, J. Tersoff, and Ph. Avouris, Deformation and Scattering in Graphene over Substrate Steps, Phys. Rev. Lett. 108, 096601 (2012).

[45] Jayakumar Balakrishnan, Gavin Kok Wai Koon, Manu Jaiswal, A. H. Castro Neto, and Barbaros Özyilmaz, Colossal enhancement of spinCorbit coupling in weakly hydrogenated graphene, Nature Phys. 9, 284 (2013).

[46] Z. F. Wang, Z. Liu, and F. Liu, Organic topological insulators in organometallic lattices, Nature Commun. 4, 1471 (2013).

[47] Z. Liu, Z.-F. Wang, J.-W. Mei, Y.-S Wu, and F. Liu, Flat Chern Band in a Two-Dimensional Organometallic Framework, Phys. Rev. Lett. 110, 106804 (2013). 